\title{
Risk factors for suicide. suicide in primary care units and in intensive care units
}

\begin{abstract}
Background: The most powerful of instincts is that of survival. The primary concern of human being is staying alive in every way. Many times it is characteristic the agonizing, people attempt survival when they faced with risks to life, serious illness, old age, even when death seems inevitable. But in all cultures and in all historical periods have been and there are still cases of people who at some point decide to terminate their existence. The purpose of this special article is to examine the risk factors such as age, race, marital status and religion-related suicides. Understanding these factors associated with suicide are deemed useful and necessary.
\end{abstract}

Methods: Literature review studies, articles have been recovered for review of computer searches. No time limit was set. Used those articles written in Greek and English.

Conclusion: The involvement of risk factors such as age, race, marital status and religion-related suicides play an important role in suicide event. Future efforts must focus on the development, prevention and treatment protocols of suicide in Primary Care. Medical and nursing staff in the Intensive Care Units must be aware of their attitudes towards the patients that have committed suicide as part of their therapeutic role.

Keywords: suicide, risk factors, primary care, intensive care unit
Volume 2 Issue 5 - 2017

\author{
M Maniou Saloustrou,' Zyga S, ${ }^{2}$ Pavlakis A, ${ }^{3}$ \\ Anathreptaki $\mathrm{AR}^{4}$ \\ 'Department of Nursing, Technological Institute of Crete, \\ Greece \\ ${ }^{2}$ Department of Nursing, University of Peloponnese, Greece \\ ${ }^{3}$ Faculty of Economics and Administration, University of \\ Neapolis Pafou, Cyprus \\ ${ }^{4}$ Department of Nursing, Technological Institute of Crete, \\ Greece
}

Correspondence: M Maniou Saloustrou, Department of Nursing, Municipality of Archanes-Asteroussia, Technological Institute of Crete, Greece, Email mmaniou@hotmail.com

Received: March 21, 2017 | Published: April 19, 2017

\section{Introduction}

The most powerful of instincts is that of survival. The primary concern of human beings and animals is staying alive in every way. Many times, it is characteristics agonizings, people attempt to survive when faced with risks to life, serious illness, old age, even when death seems inevitable. But in all cultures and in all historical periods have been and there are still cases of people who at some point decided to terminate their existence, to kill themselves.

Suicide, according to Edwin Shneidman, is the conscious selfdestruction, is not a multidimensional disorder of a person who considers this act as the best solution for an issue extremely important for him. Suicide is not an act of unnecessary or accidental. It is a way out of a problem or situation especially painful for suicide. But this way, as conflict and overcomes the fundamental instinct of survival, deviates significantly from normal human behavior. The percentage of people who eventually commit suicide is very small compared to that of people who think suicide as a solution to a difficult period of their lives.

For many centuries, the suicide phenomenon was the subject of superstitions, prejudices and prohibitions. The approach was made in ways ethical, philosophical and religious dogmatists. The first basis for scientific approach to suicide were by Durkheim, in the late 19th century, who emphasized the role played by social and cultural factors in the occurrence frequency of the phenomenon. ${ }^{1}$ In the early 20th century, Sigmund Freud was the first who make a structured psychological interpretation of suicide, with the well-known case of aggression against endostrefomenis love object. This was followed by many others, who contributed their own assumptions and opinions
(Adler, Jung, Abraham, Rado, Fenichel, Anna Freud, Horney, etc.). ${ }^{2}$

The scientific interest for suicidal behavior gradually increased and resulted in the realization of a large number of studies, as well as the appearance of Suicidology. A part from psychiatry, the scope of which are most of the studies, there has been remarkable and the contribution of other disciplines:
a. Sociology
b. Psychology
c. Anthropology
d. Epidimiologia
e. Neurobiology
f. Biochemistry
g. Genetic

According to the modern view, suicide is a complex and multifactorial human behavior, involving psychological, biological, genetic, social and cultural factors. The close relationship of suicide to the existence of psychopathology, particularly depression has been demonstrated at clinical and biological level. It is now known common disorders neurotransmitter systems, especially serotonin. From the perspective of genetic indications of a transfer increased predisposition to suicide, probably is unrelated to the transfer of mental illness. At the same time, epidemiological studies reveal significant differences in the frequency of suicide among different states and societies, thus highlighting the importance of social and cultural factors. ${ }^{3}$ 


\section{Risk factors for suicide}

\section{Gender}

Regarding gender, suicide is considered masculine, that men tend to be more suicidal than women. ${ }^{4}$ In almost all countries from which data are available, suicide indicators (MAs) are higher in men than in women (male/female ratio is 3 : of 1 to $7.5: 1$, with the exception of China) and this generally applies to all age ranges. ${ }^{5}$ A particularly interesting fact that differentiates the genders in Western countries, and that Canetto and Sakinofsky called ,gender paradox , is that women tend to often exhibit suicidal ideation and behavior (suicide attempts), however, the rates of death by suicide remains low compared to men. ${ }^{6}$ Common finding-several researchers is that the differentiation of the sexes with respect to suicidal behavior due to the influence of social and cultural factors, which several times contribute that women seek frequent medical attention, but also to choose more painlessly and thus less lethal means/methods for their self-destructive. ${ }^{7}$ Also, the same applies between prisoners, wherein the male gender is a major risk factor for suicide. ${ }^{8}$

\section{Age}

The relationship of suicide with age has been extensively studied and according to several researchers the suicide risk appears to increase with age. ${ }^{9}$ However, exceptions to this rule are constantly emerging in the literature in different times and countries. According to the mortality data by suicide of the World Health Organization (WHO) in 1995, found that female suicide indicators showed no increase with advancing age, in countries such as Mauritius, Colombia, Albania and Finland, ${ }^{10}$ while the study of Ajdacic-Gross et al. ${ }^{11}$ Covering the period from 1881 to 2000 in Switzerland, confirmed that suicide rates in both sexes increased steadily with age. Also, while in China there is an increase of suicidal indicators in both sexes with age, there was an additional peak of female suicide indices in the 15-34 age group. ${ }^{12}$

In Malaysia, according to Murty et al. ${ }^{13}$ a large percentage of the recorded suicide cases ${ }^{13}$ occurred in the age group 21-30 years, and in England and Wales, the highest ratio of male suicide occurred in the age group 25-34 years. ${ }^{14}$ In general, suicide is more common among younger age people (<45years), and increased by $44 \%$ in 1950 to $53 \%$ of total mortality in 1995 , and is due to an actual increase in the frequency of deaths by suicide in these ages, despite a reduction of suicidal acts among older age groups. However, although the suicide indicators of the elderly (>75years) remain at very high levels (almost three times higher than the equivalent of suicide indicators of persons below 25years), approximately one third of countries worldwide suicide indicators observed young people are higher than those of people of the third and fourth age. This phenomenon is recorded in countries such as Australia, Russia, Sri Lanka, Albania, Finland, but also in several islands of the Pacific Ocean, such as Western Samoa, Fiji, Micronesia, etc. ${ }^{15}$

Shah, ${ }^{16}$ in order to further investigate the relationship between suicide indicators of age took part in a transnational study (62 countries). We analyzed the mortality data from the WHO, and the main findings were that there was a statistically significant increase in suicide indexes with increasing age in men in 25 countries and women in 27 countries, and for both sexes to 17 countries. ${ }^{5}$ There was no significant increase in suicide indexes with increasing age in both men and women in 31 and 29 countries respectively for both sexes in 21 countries. ${ }^{6}$ Suicide indicators for all age groups were low in both sexes or women in those countries without a significant increase in the suicide rate with increasing age in both sexes, or women only, respectively. ${ }^{7}$ The suicide rates were higher in younger age groups in those countries without a significant increase in suicide markers in men with increasing age, and ${ }^{8}$ a group of countries observed no significant increase in suicide indexes with increasing age in both sexes (Central and South America, and Eastern Europe - countries of the former Soviet Union) only for women (South America) and for men (Eastern European countries). ${ }^{16}$ Greece showed a statistically significant increase in suicide indexes with increasing age in men, while the increase of female indicators with advancing age was not significant. This finding was confirmed in epidemiological research of Greek studies, ${ }^{17}$ while in the country recorded some variations between different regions. ${ }^{18}$

\section{Race}

Race, especially being a non-Hispanic white or Native American, is according to a recent systematic review of epidemiological surveys of Nock et al. ${ }^{19}$ a powerful risk factor for suicide. Various surveys to date mainly from the US, have found that suicide indicators of African Americans are much lower than those of white Americans, and that the former usually commit suicide at a much younger age. ${ }^{20}$ About two-thirds (2/3) of deaths from suicide in the US are committed by whites, and in countries such as Zimbabwe and South Africa, the suicide rates are higher in Caucasians than in blacks. ${ }^{15}$ Compared with other ethnic groups in the USA, Hispanic Americans are 50\% less likely to commit suicide compared with white Americans, and Asian Americans (China, Japan, Philippines) have lower suicide rates than Americans, but not as low as African Americans. ${ }^{21}$ High suicide rates have also been observed in indigenous groups (indigenous people) in the USA, Canada, Taiwan, Australia and New Zealand compared with the settlers. ${ }^{15}$ In Singapore, although as a country is a mixture of peoples and cultures, it has been observed that suicide rates of Chinese and Indian origin people are higher than those of persons originating in Malaysia. ${ }^{22}$

\section{Marital status}

As for marital status, it has been established by numerous studies that unmarried, divorced and widowed (generally people who are alone, without the presence of a partner), have higher suicide rates than married. A recent European comparative study of family status as a protective factor against socio-economic inequalities in suicide showed that the risk of suicide among unmarried persons was about two or three times higher than ${ }^{23}$ for married in many European countries. Another study, conducted in England and Wales (period 1982-1996), showed that among people who were widowed, men and women in the age group 20-39years had the highest suicide rate. ${ }^{24}$ Also, a study conducted in USA the period 1979-1981, observed that the younger widowed men (15-44 years) were the group with the highest risk of suicide compared to all other groups of unmarried,,$^{25}$ while Kposowa's study ${ }^{26}$ showed that divorced/separated men were the only group with a significantly higher risk of suicide, twice higher than married. ${ }^{26}$ In Italy, a study in a of Masocco and colleagues showed that, compared with married men, the risk of suicide was, on average, five times higher for widowers (was 13 times higher in widowers younger men) and 1.3 times higher among divorced/ separated. In women, it was observed that the risk of suicide was, on average, twice higher in unmarried women, and 1.7 times higher for widows and divorced/separated in relation to married. All, therefore, studies confirm that marriage seems to function as a protective factor against suicide, but the effect of marital status varies according to gender (men - women). ${ }^{27}$ 


\section{Socioeconomic status and occupation}

From the time of Durkheim (1897/1951), who referred to the relationship between social factors and suicide and to date, an extensive body of international literature has examined the influence of socioeconomic factors on health of the population, an inverse relationship has been observed between mortality and socioeconomic status/location (socio-economic status, SES), indicating a lower mortality from suicide in the higher socioeconomic classes regardless of socio-economic indicator used in the study or the specific cause of death. ${ }^{28}$ The relationship between the socioeconomic status of the individual and suicide is complex. The usual variables used to determine the socioeconomic status of an individual are those that reflect the social status and access to resources, such as income, education, marriage, employment and occupational status. ${ }^{29}$

The results of a recent study in Denmark (period 1981-1997) showed that suicide is closely related to a number of socioeconomic indicators, and the association is differentiated by gender. Specifically, they noticed that the poor economic situation, which is defined as lowincome, unskilled labor, unspecified paid work and unemployment, tends to increase the risk of suicide more in men than women, ${ }^{30}$ while in a study from New Zealand (period 1981-1999) ${ }^{31}$ It found that low socioeconomic class was associated with higher suicide rates across all demographic groups. Page and his colleagues ${ }^{32}$ in Australia during the period 1979-2003 found that significantly higher suicide rates among lower socio-economic classes (compared to the upper classes) were evident in both sexes throughout the period study period, while in general the men's suicide rates have increased over time in all socioeconomic classes tended to decrease in the high and middle classes and continued to rise among people

Also, Platt and Choton making a review of the literature from 1984 and since then, they argued that there is a positive correlation between low socioeconomic class and a high suicide risk. Regarding occupation, there are specific occupational categories which have been linked to increased suicide rate, such as the health professions (doctors, nurses ${ }^{33}$ farmers, ${ }^{34}$ male hunters ${ }^{35}$ students $^{36}$ and artists. ${ }^{37}$ Furthermore, Platt and Choton supports that from 1984 and since then, they argued that there is a positive correlation between low socioeconomic class and a high suicide risk. Regarding occupation, there are specific occupational categories which have been linked to increased suicide rate, such as the health professions (doctors, nurses, farmers, men hunters students and artists.

\section{Urbanization of residence}

In the international literature a common finding of researchers until the $1980 \mathrm{~s}$, was that the highest suicide rates recorded in urban and industrial areas compared to rural areas, but which contradicted by more recent longitudinal studies. ${ }^{38}$ In a recent review of the literature from 1966 to 2006, the Hirsch ${ }^{39}$ concluded that in many cases the suicide rates in rural areas is higher than in urban areas, and that rural communities present particular geographical, cultural, and socioeconomic characteristics, which possibly increase the risk of suicide. The 1970s in the US, suicide rates were lower in rural areas, while this was reversed in the 1990s and rural indicators in the last twenty years is higher $(17.9 / 100,000)$ than in urban areas $(14.9 / 100,000)$. In Canada, the suicide ratio in rural and border areas is $41 / 100,000$ population, while the total country's suicide rate is $13 / 100,000 .{ }^{39}$

In Australia, there were no significant increases in suicide rates during a 20-year (1964-1988) in urban areas, while suicide rates in rural areas doubled, and young people in rural areas increased by five times. ${ }^{38}$ Also, similar effects with higher suicide indicators in rural areas versus urban. ${ }^{39}$ have been observed in England ${ }^{40} \mathrm{Asia},{ }^{41}$ and in several other European countries ${ }^{39}$ including in Greece. ${ }^{42}$

\section{Religion}

Durkheim (1897/1951) was the first who argued that spiritual/ religious commitment contributes to emotional well-being, as it provides a source of meaning and order in the world. ${ }^{43}$ Maris, Berman, and Silverman say that religion seems to play a prominent and protective role against the decision of a person to commit a suicidal act, and that generally Protestants have higher suicide rates than Catholics, while Jews tend to be less protected from suicide than Catholics, but to a greater extent than Protestants. ${ }^{44}$ Payne, Bergin, Bielema, and Jenkins observe that religious people tend to commit suicide to a lesser extent than non-religious people, including those with a high degree of faith have a negative attitude to suicide. ${ }^{45}$ In a recent review of the literature the during period 1980 to 2008, Gearing and Lizardi examined the relationship between religion and suicide concluded that Protestants have the highest suicide rates, followed by Roman Catholics and Jews have the lowest rates. ${ }^{46}$ Also, from empirical evidence seems to suggest that the lowest rates of suicidal are among Muslims compared to other religions, such as Christianity and Hinduism. ${ }^{47}$

\section{Suicide in primary care}

Results of investigation have shown that detecting and treating depression in primary care may be effective in preventing elderly suicides, since a majority of older adults seek care services within a month of suicide. ${ }^{48}$ Prevention strategies have recommended that improved treatment and detection of depression by primary care professionals may reduce suicides among women because females are more likely to look for help in health services. ${ }^{49}$ Most primary care clinicians do not routinely screen for suicide risk. ${ }^{5} 0$ Surveys have shown that as many as $83 \%$ of suicide attempters are not identified as a danger to themselves by healthcare providers, even when examined by professionals in the months before their suicide attempt..$^{51,52}$

Consistent with men's relatively low levels of help-seeking for psychological difficulties, a review of help-seeking by individuals who eventually died by suicide showed that men had lower overall rates of contact with the formal health care system (including primary care and mental health services) compared with women. Specifically, in the year before suicide, an average $58 \%$ of women versus $35 \%$ of men sought care from a mental health practitioner. ${ }^{53}$ A program, in primary care and public health nursing, was conducted for 10 years in Matsudai town, a rural area of Japan. The results from the survey shows that female risk of completing suicide in the intervention area was reduced by $70 \%$, while there was no change in the risk for males in the intervention area for males or females in the reference area.

According to Lin et al. ${ }^{54}$ less than $20 \%$ of the suicides visited a primary care professional in the month prior to death and only $39 \%$ received treatment by specialty mental health in the 18 months prior to death. Evidence has revealed that few attempters receive care by care professionals, even after a suicide attempt. ${ }^{55}$

A survey conducted by Fordwood et al. ${ }^{56}$ showed elevated levels of psychopathology, specifically depressive symptoms, externalizing behaviors, anxiety, substance use, mania, and posttraumatic stress disorder symptoms. 
Training directors reported lack of training in depression and suicide. Remarkably, less than half of the internal pediatrics and medicine raining directors reported that training about suicide was sufficient. ${ }^{57}$ Although screening instruments have been developed for quick risk assessment, not many surveys have shown diagnostic accuracy characteristics of specificity, sensitivity, or related statistics relative to an interview with health professionals. A survey conducted showed that screening tools can identify adults and elderly in primary care who are at increased risk for suicide, although these tools produce many false-positive results. ${ }^{58}$

According to Goodwin et al. ${ }^{59}$ some physical disorders may be associated with increased suicidal ideation and may also play an important role in the relationship between suicidal ideation and depression among patients in primary care. Doctors in Primary care may wish to attend an in depth evaluation of psychiatric problems, especially current suicidal ideation, among patients with determinate physical illnesses.

\section{Suicide in intensive care unit}

Akbaba et al. ${ }^{60}$ a survey conducted in Turkey showed that suicides were $2.4 \%$ of hospital admissions (ICU and clinics). Although, the international literature supported that suicide is one of the leading causes of adolescent death. ${ }^{61}$ As far as that concern the gender and specifically for men, the main reason for admission to the ICU was car accidents $(64.4 \%)$, followed by the pathology problems $(12 \%)$, postoperative monitoring $(6.2 \%)$, work accidents $(5.6 \%)$, crimes $(5.3 \%)$, drugs (3.6\%), and finally the suicide attempt (2.9\%). ${ }^{62}$ A study conducted on the medical intensive care unit of the university hospital in Germany, between January 1993 and December 1999 showed that because of the excellent care in the prehospital phase and in the emergency room the number of patients requiring treatment on the intensive care unit was rather low. ${ }^{63}$

Mortality was in the range of other reports. According to WolkWasserman the intensive care unit's staff was interviewed about their feelings during the patients' stay on the intensive care unit. The personnel did not notice the patients' underlying feelings and primarily responded to the patients" observable behavior, ranging from distancing, aggression to empathy. Moreover, the latter reactions were due to the difficulties in dialogue between the staff and the patients which aroused anxiety and fear in the staff of the ICU. According to Wolk-Wasserman the most protrusive feelings and reactions of the patients that hospitalized in ICU were indifference and apathy in the pre psychosis/psychosis group, disgrace and resistance towards the personnel in the neurosis group and anger and uneasiness in the abuse group. ${ }^{64}$ Suokas and Lonnqvist, compared emergency department nursing staff attitudes to self-harming clients with intensive care staff and found that emergency nursing staff to be more negative towards these clients than the intensive care staff. ${ }^{65}$ The aim of a study that took place in Greece was to explore the attitudes of Greek nurses' working in surgical, medical, orthopedic, Emergency department and Intensive Care Units towards attempted suicide. Nursing staff frequently encounter suicidal patients and therefore must be aware of their attitudes towards this group of patients as part of their therapeutic role. A nurse's positive attitudes towards attempted suicide can play an important role in preventing a future suicide attempt or a fatal suicide. ${ }^{66}$

\section{Conclusion}

The involvement of risk factors such as age, race, marital status and religion-related suicides play an important role in suicide event. Many enormous steps have been made in our understanding of who is at risk for suicide. Future efforts should be done and must focus on the development, prevention and treatment protocols of suicide in Primary Care. More effective training about these vital mental health concerns in primary care could reduce mortality and morbidity. Alternate approaches to suicide prevention efforts may be needed for those less likely to be met in primary care specialty care, specifically young men. Medical and nursing staff in the Intensive Care Units must be aware of their attitudes towards the patients that have committed suicide as part of their therapeutic role. A nurse's positive attitudes towards attempted suicide can play an important role in preventing a future suicide attempt or a fatal suicide.

\section{Acknowledgements}

None.

\section{Conflict of interest}

The author declares no conflict of interest.

\section{References}

1. Stark. Rodney and William Sims Bainbridge. Religion, Deviance and Social Control. UK: Routledge; 1996

2. Sigmund Freud. The Psychopathology of Everyday Life. Brill AA editors. Toronto, USA: Springer; 1901. p. 198-206.

3. Bach $\mathrm{H}$, Arango V. Neuroanatomy of serotonergic abnormalities in suicide. In: The Neurobiological Basis of Suicide. Frontiers in Neuroscience Y Dwivedi editors. Boca Raton, Florida: Springer; 2012.

4. Nock MK, Borges G, Bromet EJ, et al. Suicide and suicidal behavior. Epidemiologic Reviews. 2008;30:133-154.

5. Hawton K, van Heeringen K. Mixed impact of firearms restrictions on fatal firearm injuries in males: a national observational study. Lancet. 2009;373:1372-1381.

6. Canetto SS, Sakinofsky I. The gender paradox in suicide. Suicide Life Threat Behav. 1998;28(1):1-23.

7. Yoshimasu K, Kiyohara C, Miyashita K. The stress research group of the japanese society for hygiene. Suicidal risk factors and completed suicide: Meta-analyses based on psychological autopsy studies. Environmental Health and Preventive Medicine. 2008;13:243-256.

8. Fazel S, Cartwright J, Norman Nott A, et al. Suicide in prisoners: A systematic review of risk factors. J Clin Psychiatry. 2008;69(11):17211731.

9. Conwell Y. Suicide prevention in later life: A glass half full, or half empty. Am J Psychiatry. 2009;166(8):845-848.

10. Shah A. The relationship between suicide rates and age: An analysis of multinational data from the World Health Organization. International Psychogeriatrics. 2007;19(6):1141-1152.

11. Ajdacic Gross V, Bopp M, Gostynski M, et al. Age-period-cohort analysis of Swiss suicide data, 1881-2000. European Archives of Psychiatry and Clinical Neuroscience. 2006;256(4):207-214.

12. Phillips MR, Li X, Zhang Y. Suicide rates in China 1995-99. Lancet 2002;359:835-840

13. Murty OP, Cheh LB, Bakit PA, et al. Suicide and ethnicity in Malaysia. American Journal of Forensic Medicine and Pathology. 2008;29:19(1)22.

14. McClure GMG. Changes in suicide in England and Wales, 1960-1997. British Journal of Psychiatry. 2000;176:64-67. 
15. De Leo D. Struggling against suicide: The need for an integrative approach. Crisis. 2002;23(1):23-31.

16. Shah A. The relationship between suicide rates and age: An analysis of multinational data from the World Health Organization. International Psychogeriatrics. 2007;19(6):1141-1152.

17. Nikolaidis G, Zavras D, Bonikos D, et al. Trends of mortality rates during the last thirty years in Greece. Journal of Medical Systems. 2004;28(6):607-616.

18. Vougiouklakis T, Boumba VA, Mitselou A, et al. Trends in suicide mortality in the deprived region of Epirus (north-west Greece) during the period 1998-2002. Medicine Science and the Law. 2005;45(1):39-46.

19. Nock MK, Borges G, Bromet EJ, et al. Suicide and suicidal behavior Epidemiologic Reviews. 2008;30(1):133-154.

20. Garlow SJ, Purselle D, Heninger M. Ethnic differences in patterns of suicide across the life cycle. American Journal of Psychiatry. 2005;162(2):319-323.

21. McKenzie K, Serfaty M, Crawford M. Suicide in ethnic minority groups. British Journal of Psychiatry. 2003;183:100-101.

22. Lorant V, Kunst AE, Huisman M, et al. The EU Working Group. A European comparative study of marital status and socio-economic inequalities in suicide. Soc Sci Med. 2005;60(11):2431-2441.

23. Yip PSF, Thorburn J. Marital status and the risk of suicide: Experience from England and Wales, 1982-1996. Psychol Rep. 2004;94(2):401-407.

24. Rosenberg ML, Davidson LE, Smith JC, et al. Operational criteria for the determination of suicide. J Forensic Sci. 1998;33(6):1445-1456.

25. Smith JC, Mercy JA, Conn JM. Marital status and the risk of suicide. Am J Public Health. 1998;78(1):78-80.

26. Kposowa AJ. Marital status and suicide in the national longitudina mortality study. J Epidemiol Community Health. 2000;54(4):254-261.

27. Masocco M, Pompili M, Vichi M, et al. Suicide and marital status in Italy. Psychiatr Q. 2008;79(4):275-285.

28. Fuhrer R, Shipley MJ, Chastang JF, et al. Socioeconomic position, health, and possible explanations: A tale of two cohorts. American Journal of Public Health. 2002;92(8):1290-1294.

29. Andrus AR, Collings S, Qin P. Sex-specific impact of socio-economic factors on suicide risk: A population-based case-control study in Denmark. Eur J Public Health. 2009;20(3):265-270.

30. Andris AR, Collings S, Qin P. Socio-economic differences in suicide risk vary by sex: A population-based case-control study of 18-65 year olds in Denmark. Ideas; 2008.

31. Collings S, Blakely T, Atkinson J, et al. Suicide trends and social factors New Zealand 1981-1999: Analyses from the New Zealand Census Mortality Study. Wellington: Ministry of Health. 2008. 67 p.

32. Page A, Morrell S, Taylor R, et al. Divergent trends in suicide by socioeconomic status in Australia. Soc Psychiatry Psychiatr Epidemiol. 2006;41(11):911-917.

33. Platt S, Hawton K. Suicidal behavior and the labour market. In: K Hawton editors. The International Handbook of suicide and attempted suicide. Chichester, UK: Springer; 2000.

34. Meltzer H, Griffiths C, Brock A, et al. Patterns of suicide by occupation in England and Wales: 2001-2005. Br J Psychiatry. 2008;193(1):73-76.

35. Skegg K, Firth H, Gray A,et al. Suicide by occupation: Does access to means increase the risk. Aust N Z J Psychiatry. 2010;44(5):429-434.

36. Collins IP, Paykel ES. Suicide amongst Cambridge University students 1970-1996. Social Psychiatry and Psychiatric Epidemiology. 2000;35(3):128-132.
37. Stack S. Gender and suicide risk among artists: A multivariate analysis. Suicide and Life-Threatening Behavior. 1996;26(4):374-379.

38. Kapusta ND, Zorman A, Etzersdorfe E, et al. Rural-urban differences in Austrian suicides. Soc Psychiatry Psychiatr Epidemiol. 2008;43(4):311318.

39. Hirsch JK. A review of the literature on rural suicide: Risk and protective factors, incidence, and prevention. Crisis. 2006;27(4):189-199.

40. Middleton N, Gunnell D, Frankel S, et al. Urban-rural differences in suicide trends in young adults: England and Wales, 1981-1998. Soc Sci Med. 2003;57(7):1183-1194.

41. Pritchard C. Suicide in the People's Republic of China categorized by age and gender: Evidence of the influence of culture on suicide. Acta Psychiatr Scand. 1996;93(5):362-367.

42. Beratis S. Suicide in southwestern Greece 1979-1984. Acta Psychiatr Scand. 1986;74(5):433-439.

43. Durkheim E. Suicide: A study in sociology. New York, USA: The Free Press; 1951.

44. Maris RW, Berman AL, Silverman MM. Comprehensive textbook of suicidology. New York, US: Guilford Press; 2000.

45. Payne IR, Bergin AE, Bielema KA, et al. Review of religion and mental health: Prevention and the enhancement of psychosocial functioning. In: KI Pargament editors. Religion and prevention in mental health: Research, vision and action. Binghamton, USA: Haworth Press; 1992. p. $57-82$.

46. Gearing RE, Lizardi D. Religion and suicide. $J$ Relig Health 2009;48(3):332-341

47. Lizardi D, Gearing RE. Religion and suicide: Buddhism, Native American and African religions, Atheism, and Agnosticism. J Relig Health. 2010;49(3):377-384.

48. Clark D. Suicidal behavior in childhood and adolescence: recent studies and clinical implications. Psychiatr Ann. 1993;23(5):271-283.

49. Rutz W, von Knorring L, Pihlgren H, et al. An educational project on depression and its consequences: is the frequency of major depression among Swedish men underrated, resulting in high suicidality. Primary Care Psychiatry. 1995;1:59-63.

50. Olson AL, Gaffney CA, Hedberg VA, et al. Use of inexpensive technology to enhance adolescent health screening and counseling. Arch Pediatr Adolesc Med. 2009;163(2):172-177.

51. Frankenfield DL, Keyl PM, Gielen A, et al. Adolescent patients: healthy or hurting? Missed opportunities to screen for suicide risk in the primary care setting. Arch Pediatr Adolesc Med. 2000;154(2):162-168.

52. Oyama H, GotoRelated M, FujitaRelated M, et al. Preventing Elderly Suicide Through Primary Care by Community-Based Screening for Depression in Rural Japan. Crisis. 2006;27(2):58-65.

53. Eaton DK, Kann L, Kinchen S, et al. Youth risk behavior surveillanceUnited States, 2007. MMWR Surveill Summ. 2008;57:1-131.

54. Lin E, Michael Von Korff, Wagner E. Identifying suicide potential in primary care. Journal of General Internal Medicine. 1989;4(1):1-6.

55. Conwell Y, Lyness M, Duberstein P, et al. Completed Suicide Among Older Patients in Primary Care Practices: A Controlled Study. Journal of the American Geriatrics Society. 2000;48(1):23-29.

56. Fordwood S, Asarnow J, Huizar D, et al. Suicide Attempts Among Depressed Adolescents in Primary Care. Journal of Clinical Child \& Adolescent Psychology. 2007;36(3).

57. Sudak D, Sudak H, Lipschitz A, et al. Deficiencies in Suicide Training in Primary Care Specialties: A Survey of Training Directors. Academic Psychiatry. 2007;31(5):345-349. 
58. Gaynes B, West S, Ford CA, et al. Screening for Suicide Risk in Adults A Summary of the Evidence for the U.S. Preventive Services Task Force Ann Intern Med. 2004;140(10):822-835.

59. Goodwin D, Kroenke K, Hoven CW, et al. Major depression, physical illness, and suicidal ideation in primary care. Psychosom Med. 2003;65(4):501-505.

60. Akbaba M, Nazlican E, Demirhindi $H$, et al. Etiological and demographical characteristics of acute adult poisoning inAdana, Turkey. Human \& Experimental Toxicology. 2007;26(5):401-406.

61. Moskos MA, Achilles J, Gray D. Adolescent suicide myths in the United States. Crisis. 2004;25(4):176-182

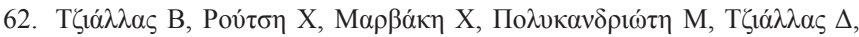

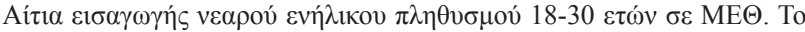

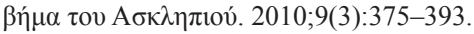

63. Viertel A, Weidmann E, Rbrodt H. Cases of acute poisoning admitted to a medical intensive care unit. Dtsch med Wochenschr. 2001;126(42):11591163.

64. Wolk Wasserman D. The intensive care unit and the suicide attempt patient. Acta Psychiatr Scand. 1985;71(6):581-595.

65. Suokas J, Lonnqvist J. Work stress has negative effects on the attitudes of emergency personnel towards patients who attempt suicide. Acta Psychiatr Scand. 1989;79(5):474-480.

66. Ouzouni C, Nakakis K. Nurses' attitudes towards attempted suicide. Health Science Journal. 2013;7(1):119-134. 